\title{
Active Gravitational Mass and The Invariant Characterization of Reissner-Nordström Spacetime
}

\author{
L. Herrera ${ }^{1 *}$ N. O. Santos ${ }^{2,3 \dagger}$ and J. E. F. Skea ${ }^{4 \dagger}$ \\ ${ }^{1}$ Escuela de Física, Facultad de Ciencias, \\ Universidad Central de Venezuela, Caracas, Venezuela. \\ ${ }^{2}$ Laboratório Nacional de Computa cão Científica, \\ 25651-070 Petrópolis RJ, Brazil. \\ ${ }^{3}$ Centro Brasileiro de Pesquisas Físicas, \\ 22290-180 Rio de Janeiro RJ, Brazil. \\ ${ }^{4}$ Departamento de Física Teórica, \\ Universidade Estadual do Rio de Janeiro, \\ 20550-013 Rio de Janeiro RJ, Brazil. \\ June 10, 2018
}

\begin{abstract}
We analyse the concept of active gravitational mass for ReissnerNordström spacetime in terms of scalar polynomial invariants and the Karlhede classification. We show that while the Kretschmann scalar does not produce the expected expression for the active gravitational mass, both scalar polynomial invariants formed from the Weyl tensor, and the Cartan scalars, do.
\end{abstract}

*e-mail: laherrera@telcel.net.ve

†e-mail: nos@cbpf.br

†e-mail: jimsk@dft.if.uerj.br 


\section{Introduction}

In a recent paper [1] the Kretschmann scalar is attributed with the role of characterizing the curvature of spacetime: in the words of the author, allowing one to "see" the black hole and so any possible black hole can be visualized realistically. Besides this scalar, the scalars of Chern-Pontryagin and Euler have also been studied [2] in a similar context. In this letter we look at the invariant characterization of the curvature of spacetime using a different approach.

We restrict ourselves to spherical symmetry and to the analysis of the Reissner-Nordström (RN) spacetime $[3,4]$

$$
\mathrm{d} s^{2}=A \mathrm{~d} t^{2}-A^{-1} \mathrm{~d} r^{2}-r^{2}\left(\mathrm{~d} \theta^{2}+\sin ^{2} \theta \mathrm{d} \phi^{2}\right),
$$

where $A$ is defined by

$$
A \equiv 1-\frac{2 M}{r}+\frac{Q^{2}}{r^{2}},
$$

with $M$ the mass parameter and $Q$ the charge.

By taking a spherical surface, $\Sigma$, centred at $r=0$ in the coordinate system describing the metric (1) we expect that the gravitational field outside $\Sigma$ does not affect the field inside $\Sigma$. The mass $M$ as well as the charge $Q$ produce gravitational field. Hence it is reasonable to expect that we can find a quantity at $r$, that we call gravitational mass $m(r)$, that takes the place of $M$ and $Q$ by producing a corresponding Schwarzschild (S) gravitational field [5] at $\Sigma$. Our approach to find an eligible invariant expression to describe physically the curvature should be one that reproduces the corresponding $\mathrm{S}$ expression with $m(r)$.

\section{The active gravitational mass}

In a recent paper [6] different concepts of gravitational mass for the RN spacetime are discussed. The favoured definition, by the authors, is the active gravitational mass, $m_{a}(r)$, obtained by Whittaker [7]. Starting from the active gravitational mass density, $\mu$, defined by Whittaker [7] and Tolman [8] as

$$
\mu=E_{0}^{0}-E_{i}^{i}
$$


where $E_{\beta}^{\alpha}$ is the electromagnetic energy tensor, the active gravitational mass inside a volume $V$ is given by

$$
m_{a}(r)=\int_{V} \mu(-g)^{1 / 2} \mathrm{~d} x^{1} \mathrm{~d} x^{2} \mathrm{~d} x^{3}
$$

where $g$ is the four-dimensional determinant of the metric. Applying (4) to the metric (1) we find

$$
m_{a}(\infty)-m_{a}(r)=\int_{r}^{\infty} \frac{Q^{2}}{r^{2}} \mathrm{~d} r
$$

Since $M$ prevails asymptotically in RN spacetime we assume $m_{a}(\infty)=M$, and (5) becomes

$$
m_{a}(r)=M-\frac{Q^{2}}{r} .
$$

Considering a general anisotropic charged static fluid source for the RN spacetime it is possible to prove [9] that, if the energy conditions are satisfied, the maximum charge allowed inside the fluid sphere of boundary radius $r_{b}$, is

$$
Q_{\max }^{2}=M r_{b}
$$

Another interpretation [9] of (7), is that the energy conditions impose a lower limit for the size of the fluid distribution,

$$
r_{b_{\min }}=\frac{Q^{2}}{M}
$$

Hence, condition (8) imposes on (6) that the active gravitational mass $m_{a} \geq$ 0 .

Some further reasons that suggest that $m_{a}(r)$ is a plausible definition are the following. The equations governing the radial geodesics and the circular geodesics in the equatorial plane of RN spacetime for a chargeless test particle are, respectively,

$$
\frac{\mathrm{d}^{2} r}{\mathrm{~d} \tau^{2}}=-\frac{m_{a}(r)}{r^{2}}, \quad\left(\frac{\mathrm{d} \phi}{\mathrm{d} \tau}\right)^{2}=\frac{m_{a}(r)}{r^{3}}
$$

where $\tau$ is the proper time. We see from (9) that locally $m_{a}(r)$ casts the equation of motion in a Newtonian like form. Another reason is that at 
the event horizon of RN spacetime the active gravitational mass is equal to the geometrical S mass of the RN spacetime [6]. Furthermore, as shown in [10], the active gravitational mass allows one to obtain a better grasp of the physical content of the matter when analysing the energy content of a slowly collapsing gravitating sphere.

\section{The Weyl and Cartan scalars}

As is well known, the Riemann curvature tensor can be separated in a coordinate invariant way into the Weyl tensor, the Ricci tensor and the curvature scalar, from which we may deduce that the Weyl is generated only by the gravitational field. From the Weyl tensor we may construct the Weyl scalar $\mathcal{C} \equiv C^{\alpha \beta \gamma \delta} C_{\alpha \beta \gamma \delta}$, which, for the RN spacetime (1), is

$$
\mathcal{C}_{\mathrm{RN}}=\frac{48}{r^{6}}\left(M-\frac{Q^{2}}{r}\right)^{2} .
$$

Comparing (10) with the corresponding form for the Schwarzschild spacetime with mass parameter $m$,

$$
\mathcal{C}_{\mathrm{S}}=\frac{48}{r^{6}} m^{2}
$$

we see that the expressions are equivalent if we make the identification (6) for $m_{a}(r)$.

Another way of describing a spacetime invariantly is by its Karlhede classification [11]. Using a basis fixed up to the isotropy group of the spacetime, the frame components of the decompositions of the Riemann tensor and its covariant derivatives become invariantly defined scalars for the spacetime. These scalars have been referred to in the literature [12] as the Cartan scalars for the spacetime. The Cartan scalars provide a more refined invariant characterization of the spacetime than scalar polynomial invariants since, for example, they distinguish between the Minkowski spacetime and special plane wave solutions, for which all scalar polynomial invariants vanish. Notice that although Cartan scalars transform like scalars under coordinate transformations, they transform like spinor components under basis transformations

In spinor notation, one of the Cartan scalars for the RN spacetime is the 
only non-null component of the Weyl spinor

$$
\left(\Psi_{2}\right)_{\mathrm{RN}}=-\frac{1}{r^{3}}\left(M-\frac{Q^{2}}{r}\right),
$$

For the Schwarzschild spacetime with mass parameter $m$ the corresponding Cartan scalar is

$$
\left(\Psi_{2}\right)_{\mathrm{S}}=-\frac{m}{r^{3}}
$$

and once again we identify equivalent forms if we use the definition (6) for the gravitational mass. Several authors [13], [14] associate $\Psi_{2}$ with the purely gravitational energy that arises from the Weyl tensor for a collapsing fluid sphere.

In passing we note that the Kretschmann scalar $\mathcal{R}=R^{\alpha \beta \gamma \delta} R_{\alpha \beta \gamma \delta}$ for the $\mathrm{RN}$ spacetime is

$$
\mathcal{R}_{R N}=\frac{46}{r^{6}}\left[\left(M-\frac{Q^{2}}{r}\right)^{2}+\frac{Q^{4}}{6 r^{2}}\right],
$$

which cannot be written in the corresponding Schwarzschild form with $m_{a}(r)$. Returning to the beginning of this article, while this scalar may be useful for measuring curvature near a Schwarzschild black hole, it does not seem to us to be the case that with it "any possible black hole can be visualized realistically". The Weyl and Cartan scalars seem to be better suited for this purpose in the non-vacuum cases.

\section{References}

[1] Henry R C, Ap. J. 535, 350 (2000)

[2] Cherubini C, Bini D, Capozziello S and Ruffini R, Int. J. Mod. Phys. D11, 827 (2002)

[3] Reissner H, Annal. d. Phys. 50, 106 (1916)

[4] Nordström G, Proc. Kon. Ned. Akad. Wet. 20, 1238 (1918)

[5] Schwartzschild K. Sitz. Preuß. Akad. Wiss. 189 1916; English translation see: Gen. Rel. Grav. 35 (2003) 951 
[6] Barbachoux C, Gariel J, Marcilhacy G and Santos N O, Int. J. Mod. Phys. D11, 1255 (2002)

[7] Whittaker E T, Proc. Roy. Soc. London A149, 384 (1935)

[8] Tolman R, Phys. Rev. 35, 875 (1930)

[9] Ponce de Leon J, Gen. Rel. Grav. 25, 1123 (1993)

[10] Herrera L and Santos N O, Gen. Rel. Grav. 27, 1071 (1995)

[11] Karlhede A, Gen. Rel. Grav. 12, 693 (1980)

[12] da Silva M F A, Herrera L, Paiva F M and Santos N O, Gen. Rel. Grav. 27, 859 (1995)

[13] Szekeres P, J. Math. Phys. 61387 (1965)

[14] Glass E N, J. Math. Phys. 20, 1508 (1979) 